\title{
Eine Reformulierung des Diskriminationsmodells ${ }^{1}$
}

\author{
Norman Braun
}

Universität Bern, Institut für Soziologie, Lerchenweg 36, CH-3000 Bern 9

Zusammenfassung: Dieser Artikel modifiziert und erweitert Lindenbergs Diskriminationsmodell. Letzteres wird oftmals als Modell für begrenzt rationale Handlungsentscheidungen interpretiert, die sich im Gefolge kognitiver Kapazitätsgrenzen ergeben. Seine Reformulierung beseitigt die formalen Schwächen des Originals. Aufgrund der dafür notwendigen Annahmen über die menschlichen Fähigkeiten bei der Aufnahme und Verarbeitung von Informationen scheint das Diskriminationsmodell jedoch für die Abbildung begrenzter Rationalität ebensowenig geeignet wie der (als Grenzfall resultierende) Erwartungsnutzenansatz.

\section{Einführung}

In der Literatur zum menschlichen Entscheidungsverhalten wird immer wieder betont, dass die Annahme der begrenzten Rationalität dem Postulat vollkommener Rationalität (z.B. Maximierung des (erwarteten) Nutzens) vorzuziehen ist. Allerdings werden dem Begriff der begrenzten Rationalität dabei verschiedene Bedeutungen zugewiesen. In der Interpretation von z. B. Williamson (1985: 46) wird begrenzte Rationalität als unvollständige Information konzeptualisiert, die gegebenenfalls vollkommen rational verwertet wird. Im Gegensatz dazu argumentiert z. B. Selten (1990: 657), dass begrenzte Rationalität nicht bloss als eine modifizierte Nutzenmaximierung aufgefasst werden kann. Nach Simon (z.B. 1990: 15) bezeichnet begrenzte Rationalität mehr oder weniger zielgerichtetes Verhalten, das kognitive Kapazitätsgrenzen (z.B. Mängel der Aufmerksamkeitslenkung, Probleme der Informationsverarbeitung) reflektiert. Demnach liegt begrenzte Rationalität u.a. dann vor, wenn (gegebenenfalls vollständige) Information aufgrund von Kapazitätsbeschränkungen unvollkommen verarbeitet wird.

Diese Interpretationen schlagen sich in verschiedenen Ansätzen zur Modellierung begrenzt rationalen Verhaltens nieder. In der Ökonomik liegen diverse Modellierungsvorschläge vor, deren Unterschiede daneben durch Kennzeichen der jeweils betrachteten Entscheidungssituation bedingt sind (z. B. Kreps 1990; Radner 1980; Selten 1977; Simon 1982; Tietz/Albers/Selten 1988). Auch in der Soziologie existieren inzwischen Vorschläge zur

${ }^{1}$ Für hilfreiche Anregungen und Verbesserungsvorschläge danke ich Henriette Engelhardt, Axel Franzen, Thomas Gautschi, Claudia Zahner sowie - für die ZfS - Hartmut Esser und Werner Raub.
Modellierung begrenzt rationaler Handlungswahlen: Während Esser (z.B. 1993) begrenzt rationales Verhalten im Sinne einer zweistufigen Erwartungsnutzenentscheidung konzeptualisiert, formuliert Lindenberg (z. B. 1993) sein Diskriminationsmodell (DM) ohne expliziten Rückgriff auf die Annahme der Erwartungsnutzenmaximierung. Begrenzte Rationalität wird dabei als begrenzte Kapazität bei der Verarbeitung von (gegebenenfalls vollständiger) Information gedeutet.

Lüdemann/Rothgang (1996) diskutieren Stärken und Schwächen der Modelle von Esser und Lindenberg. Dabei betonen sie insbesondere die Notwendigkeit weiterer Arbeit an Lindenbergs Theorie. Nach der Diagnose von Lüdemann/Rothgang (1996: 287) bietet das DM in seiner derzeitigen Form noch keine Möglichkeit, „... die Wahl eines dominierenden Handlungsziels und die Entscheidung über eine Handlung im Hinblick auf dieses Ziel angemessen zu modellieren.“

Der vorliegende Artikel reformuliert das DM im Sinne der grundlegenden Ideen Lindenbergs, um dieses Problem zu lösen. Bevor die Erweiterung und Modifikation des DM vorgestellt wird, erscheint eine knappe Beschreibung des Originalmodells sinnvoll. Abschliessend wird diskutiert, ob das DM als Modell für begrenzt rationales Verhalten angesehen werden kann.

\section{Diskriminationsmodell}

Lindenberg (1993) betrachtet einen einzigen Akteur, der mit einer endlichen Zahl diskreter Handlungsalternativen $(i=1,2, \ldots n)$ konfrontiert ist, die jeweils potentiell unsichere Handlungskonsequenzen nach sich ziehen. Diese Person hat exogen vorgegebene Präferenzen für verschiedene fundamentale Ziele (z.B. physisches Wohlbefin- 
den, soziale Wertschätzung). Nach Lindenberg kann sie aufgrund kognitiver Limitationen lediglich ein allgemeines Ziel bei ihrer Handlungsentscheidung verfolgen. Nur ein einziges ausgewähltes Ziel liefert somit den Bezugsrahmen (,frame“) für die Handlungsentscheidung. Die Dominanz dieses Rahmens in der Entscheidungssituation wird durch einen situationsbezogenen Gewichtungsparameter („situational salience“) gemessen, welcher (in nicht näher spezifizierter Form) von den allgemeinen Zielen abhängt. Vor dem Hintergrund des Frames und des Dominanzparameters wird dann die Handlungsentscheidung getroffen. Damit ist das DM ein zweistufiges Entscheidungsmodell:

- Zunächst wählt der Akteur ein handlungsleitendes Ziel und damit einen Frame aus einer Menge möglicher Bezugsrahmen, wobei durch die allgemeinen Ziele die situationale Dominanz des gewählten Rahmens determiniert wird.

- Vor dem Hintergrund dieses Handlungsziels und dem Ausmass seiner Dominanz in der betrachteten Entscheidungssituation bestimmt der Akteur dann die Auswahlwahrscheinlichkeit für jede Handlungsalternative und führt schliesslich diejenige Handlung aus, welche dabei die höchste Auswahlwahrscheinlichkeit aufweist.

Da diese Handlungsentscheidung innerhalb eines bestimmten Frames stattfindet, wählt der Akteur nicht unbedingt diejenige Alternative, die den insgesamt höchsten Erwartungsnutzen stiftet. Aufgrund dieser potentiellen Abweichung vom traditionellen Erwartungsnutzenmodell interpretieren Lindenberg sowie Lüdemann/Rothgang (1996) das DM als ein Modell der begrenzten Rationalität.

Wie Lüdemann/Rothgang allerdings auch betonen, handelt es sich bei der von Lindenberg bis dato formulierten Version des DM um kein vollständig ausgearbeitetes Modell. Lindenberg verzichtet nämlich auf die Modellierung der Wahl eines Frames und die Endogenisierung seiner situationalen Dominanz. Er konzentriert sich stattdessen auf die Bestimmungsgrössen der Wahlwahrscheinlichkeit $w_{i}$ für jede Handlungsalternative $i$ innerhalb eines bereits gegebenen Frames. Diese modellexogenen Determinanten sind

- die Abweichung von $u_{i}$, dem erwarteten Nutzen der $i$-ten Handlungsalternative, vom durchschnittlichen Erwartungsnutzen $\bar{u}=(1 / n) \sum u_{i}$ im aktuellen Frame sowie

- der Betonungs- oder Gewichtungsparameter $\beta$ für die situationale Dominanz des gewählten Bezugsrahmens.
Lindenbergs Modellierungsvorschlag für die Bestimmung der Handlungsalternative innerhalb eines gegebenen Frame beruht auf den zusätzlichen Annahmen $0 \leq \beta \leq 1$ und $0 \leq u_{1} \leq 1$ für alle $i$. Damit werden sowohl der Gewichtungsparameter als auch die Erwartungsnutzenwerte offenbar auf Verhältnisskalenniveau gemessen. Die Wahlwahrscheinlichkeit $w_{i}$ jeder Handlungsalternative $i$ wird ebenfalls durch eine Annahme festgelegt:

$w_{1}=(1 / n)+(1 / n) \beta\left(u_{1}-\tilde{u}\right)$ für alle $i$.

Nach der Bestimmung sämtlicher Wahlwahrscheinlichkeiten führt der Akteur dann diejenige Handlungsalternative aus, welche innerhalb des aktuellen Frames die höchste Wahlwahrscheinlichkeit aufweist. Diese Alternative lässt sich im dominierenden Bezugsrahmen als die am stärksten diskriminierende Handlungsalternative interpretieren.

Da Lüdemann/Rothgang (1996) ausfuhrlich auf formale und inhaltliche Details von Lindenbergs Modell eingehen, kann hier auf eine genauere Diskussion dieser Aspekte verzichtet werden. Die Hauptkritikpunkte betreffen

- die unklaren Bedingungen bei der Wahl des jeweils relevanten Bezugsrahmens,

- die fehlende Modellierung für einen Wechsel des Rahmens (,frame switch") sowie

- die Exogenität des situationsbezogenen Gewichtungsparameters (d.h. der situationalen Dominanz des gewählten Frames).

Die folgende Reformulierung des DM kann als Versuch gedeutet werden, diese Mängel im Sinne von Lindenbergs grundlegenden Ideen zu beseitigen. Trotzdem sind für die Formalisierung einige Reinterpretationen und Spezifikationen unvermeidlich, die nicht unbedingt Lindenbergs Intentionen entsprechen müssen.

\section{Reformuliertes Modell}

\subsection{Konzepte und Grundideen}

Betrachtet wird ein typischer Akteur in einer diskreten Entscheidungssituation. Letztere ist charakterisiert durch eine endliche Zahl von unterschiedlichen Handlungsalternativen $(i=1,2, \ldots n)$ mit jeweils $r_{i}$ erschöpfenden und sich gegenseitig ausschliessenden Handlungskonsequenzen. Vor seiner Handlungsentscheidung hat der Akteur die Wahl zwischen verschiedenen Handlungsorientierungen $(j=1,2, \ldots, m)$, die jeweils das Leitmotiv der Handlungsentscheidung sein können. Der Be- 
griff der Handlungsorientierung ersetzt hier simultan Lindenbergs Begriffe des Hintergrundziels und des Bezugsrahmens. Diese Ersetzung ist aus mehreren Gründen sinnvoll: Die Grundideen des DM werden durch sie nicht in Frage gestellt, unscharfe Begriffe sollten bei der Theoriebildung möglichst reduziert werden, die Heterogenität von Entscheidungssituationen verhindert ohnehin eine verbindliche Liste der Hintergrundziele und Bezugsrahmen und, last but not least, für die Modellierung der zentralen Ideen Lindenbergs genügt das einzelne Konzept der Handlungsorientierung.

Mit dieser Begriffsersetzung ist das u.a. von Lüdemann/Rothgang (1996) angesprochene Problem der fehlenden Festlegung von Hintergrundzielen und Bezugsrahmen im DM natürlich nicht gelöst, sondern nur auf eine andere Ebene geschoben. Bei konkreten Anwendungen des DM ist es nach wie vor erforderlich, die einzelnen Handlungsorientierungen zu spezifizieren. Eine naheliegende Spezifikation aus soziologischer Sicht könnte z. B. Webers (1921) idealtypische Unterscheidung von zweckrationalem Handeln, wertrationalem Handeln, affektuellem Handeln und traditionalem Handeln sein. Jedoch sind auch alternative Klassifikationen von Handlungsorientierungen denkbar. Alternativ könnte man, etwa im Gefolge Etzionis (1994), Egoismus und Moralität als grundlegende Handlungsmotivationen betrachten. Diese Beispiele verdeutlichen, dass die Zahl von Orientierungen möglichst gering zu halten ist. Verschiedene Handlungsorientierungen gehen typischerweise nämlich mit unterschiedlichen Wertvorstellungen (und daher Nutzenfunktionen) einher, so dass Sparsamkeit bei der Wahl der Grundmotive eine wichtige Voraussetzung für die empirische Anwendbarkeit des DM darstellt.

Die Verwendung des zusammenfassenden Konzepts der „Handlungsorientierung" führt zu einer leichten Umformulierung der Grundideen Lindenbergs. So wird der Dominanzgrad einer zugrundegelegten Handlungsorientierung jetzt von exogen vorgegebenen Präferenzen des Akteurs für die einzelnen Handlungsleitmotive bestimmt. Die Handlungsentscheidungen beruhen dann auf exogenen Nutzenwerten, die sich vor dem Hintergrund der gerade aktuellen Handlungsorientierung ergeben. $\mathrm{Zu}$ endogenisieren sind die jeweils vorherrschende Handlungsorientierung, der $\mathrm{Ge}-$ wichtungsparameter für ihre situationale Dominanz sowie die Wahlwahrscheinlichkeiten der Handlungsalternativen für jede mögliche Handlungsorientierung.

\subsection{Dominanz der Handlungsorientierung}

Angenommen wird, dass der Akteur exogen vorgegebene und hinreichend stabile Präferenzintensitäten für verschiedene Handlungsorientierungen besitzt. Diese Präferenzen über grundlegende Handlungsmotive kann man als Resultat der persönlichen Erfahrungen und sozialen Einflüsse vor der eigentlichen Entscheidungssituation deuten. Seien $x_{1}, x_{2}, \ldots x_{m}$ die Interessengrade an den insgesamt $m$ unterschiedlichen Orientierungen, so dass

$x_{j} \geq 0$ für alle $j$ und $\sum_{j=1}^{m} x_{j}=1$.

Damit wird die Messung von Präferenzintensitäten auf Absolutskalenniveau verlangt. Da Lindenberg aber ohnehin bereits Verhältnisskalen für die Nutzenmessung unterstellt, erscheint die Annahme der Interessennormierung wenig problematisch. Eine derartige Annahme ist im übrigen bei soziologischen Anwendungen der Theorie rationalen Handelns keineswegs ungewöhnlich (z.B. Coleman 1990).

Bei gleichmässiger verteilten Interessen an den einzelnen Handlungsorientierungen wird die situationale Dominanz einer gewählten Orientierung in der betrachteten Entscheidungssituation geringer sein. Um diese Idee zu modellieren, benötigt man ein Kriterium zur vergleichenden Beurteilung der Elemente einer Verteilung. Ein einfaches und gleichzeitig axiomatisch begründbares Kriterium erhält man bei Verwendung des Entropiekonzeptes aus der Informationstheorie (z.B. Maasoumi 1990). Die Entropie gibt das Ausmass der Unordnung bzw. Unsicherheit in einem geschlossenen System an. Sie ergibt sich in unserem Zusammenhang als

$F(\mathrm{x})=-\sum_{j=1}^{m} x_{j} \ln x_{j}$

Weil $x_{j} \ln x_{j} \rightarrow 0$ für $x_{j} \rightarrow 0$ gilt, wird die Konvention $x_{j} \ln x_{j}=0$ für $x_{j}=0$ eingeführt. Aufgrund des Definitionsbereiches der Interessengrade besitzt die Entropiefunktion $F(\cdot)$ dann den Wertebereich $[0, \ln m]$. Dabei führt Gleichheit sämtlicher Interessengrade (d.h. $x_{j}=(1 / m)$ für alle $j$ ) zur maximalen Entropie, während die extremste Ungleichheit der Interessen (d.h. $x_{j}=1$, aber $x_{h}=0$ für alle $h \neq j$ ) die minimale Entropie erzeugt. Da das globale Maximum der Entropie an der Stelle der Gleichverteilung liegt und $\ln m$ den korrespondierenden Maximalwert der Entropie festlegt, misst $F(\mathrm{x})$ die Abweichung der tatsächlichen Verteilung vom Szenario der Gleichverteilung. 
Man kann deswegen die Ungleichheit der beobachteten Verteilung durch die Differenz zwischen dem Maximalwert der Entropie und der tatsächlichen Entropie erfassen (Theil 1967). Die Normalisierung dieser Ungleichheitsmessung mit der maximalen Entropie führt zu Colemans (1964) Hierarchieindex, der generell den Grad der Differenzierung in einem geschlossenen System angibt. In unserem Zusammenhang bestimmt Colemans Index die Hierarchisierung der Präferenzintensitäten für Handlungsmotive. Er kann daher im Sinne von Lindenberg als der Grad der situationalen Dominanz einer gewählten Handlungsorientierung interpretiert werden:

$\beta=H(\mathrm{x})=\frac{\ln m-F(\mathrm{x})}{\ln m}=1+\frac{\sum_{1} x_{1} \ln x_{j}}{\ln m}$,

so dass $0 \leq \beta \leq 1$. Somit ist $\beta=0$, wenn $x_{j}=(1 / m)$ für alle $j$ sowie $\beta=1$, falls $x_{j}=1$ und $x_{h}=0$ für alle $h \neq$ $j$. Je gleichmässiger die Interessen an den Handlungsmotiven verteilt sind, desto geringer ist für den Akteur in der betrachteten Entscheidungssituation die situationale Dominanz einer zugrundegelegten Handlungsorientierung.

\subsection{Wahlwahrscheinlichkeiten}

Sei $v_{j i}$ der normierte Erwartungsnutzen (oder die Präferenzintensität bezüglich) der $i$-ten Handlungsalternative innerhalb der $j$-ten Handlungsorientierung:

$v_{j i} \geq 0$ für alle $j, i$ und $\sum_{i=1}^{n} v_{j i}=1$ für alle $j$.

Auch diese Annahme stellt nur eine leichte Erweiterung von Lindenbergs Ansatz dar, da dieser ohnehin Ratioskalenniveau bei der Nutzenmessung unterstellt. Die Normierung der Erwartungsnutzenwerte bedeutet, dass der Akteur für eine gewählte Handlungsorientierung ein fixes Gesamtinteresse besitzt, das er oder sie auf sämtliche Entscheidungsalternativen streut. Eine sofortige Konsequenz dieses Postulats ist, dass der Durchschnittsnutzen der Alternativen für jede beliebige Handlungsorientierung gleich ist:

$\bar{v}_{j}=(1 / n) \sum_{i=1}^{n} v_{j i}=(1 / n)$ für alle $j$.

Die Standardisierung der Interessen an Handlungsalternativen legt somit einen eindeutigen $\mathrm{Re}$ ferenzpunkt für alle möglichen Handlungsorientierungen fest.

Abweichend von Lindenbergs Ansatz sei die Auswahlwahrscheinlichkeit der $i$-ten Alternative vor dem Hintergrund von Leitmotiv $j$ ein gewichteter Durchschnitt aus $v_{j i}$, ihrem normierten Erwartungsnutzen, und $\bar{v}_{j}=(1 / n)$, dem Durchschnitt der skalierten Erwartungsnutzenwerte:

$w_{j}=\beta v_{j i}+(1-\beta) \bar{v}_{j}=(1 / n)+\beta\left(v_{j i}-(1 / n)\right)$

für alle $j, i$,

so dass sich aufgrund der Endogenisierung des Dominanzparameters $\beta$ folgender Ausdruck ergibt:

$w_{j i}=\frac{v_{\mu} \ln m+\left(v_{\mu}-(1 / n)\right) \sum_{h=1}^{m} x_{h} \ln x_{h}}{\ln m}$ für alle $j, i$.

Es gilt damit $w_{j i} \geq 0, \sum_{k=1}^{n} w_{j k}=1$ und daher auch $w_{j i}$ $\leq 1$ für alle $j, i$. Bei Gleichheit aller $v_{j i}$ für die Orientierung $j$ gilt weiter, dass $v_{i i}=(1 / n)=w_{j i}$ für alle $i$. Zudem ergibt sich bei $v_{j i}=1$ und $v_{j k}=0$ für alle $k \neq$ $i$, dass $w_{j i}=\beta+(1-\beta) / n$ und $w_{j k}=(1-\beta) / n$ für alle $k \neq i$. Ist der Akteur nur an der Handlungsorientierung $j$ (also an den anderen $m-1$ Handlungsmotiven nicht) interessiert, so konvergiert der situationale Dominanzparameter $\beta$ gegen Eins und die Wahlwahrscheinlichkeit der $i$-ten Handlungsalternative reduziert sich auf $w_{j i}=v_{j i}$. Bezeichnet man mit $u_{j}$ den Erwartungsnutzen bezuglich der $i$-ten Handlungsalternative bei Handlungsorientierung $j$ $\left(0 \leq u_{\mu} \leq 1\right)$, dann ist der normierte Erwartungsnutzenwert $v_{j i}$ definiert durch

$v_{j i}=\frac{u_{j i}}{\sum_{k=1}^{n} u_{j k}}$ für alle $j, i$,

so dass für $\beta=1$ individuelles Wahlverhalten im Sinne des Erwartungsnutzenansatzes auftritt. Erwartungsnutzenmaximierung kann daher als Grenzfall des reformulierten Modells angesehen werden. Somit lassen sich die (u.a. von Lüdemann/ Rothgang (1996) beklagten) formalen Probleme des DM lösen.

Daneben resultiert durch die (im Gegensatz zum Original) unterstellte Durchschnittsbildung bei der Bestimmung der Wahlwahrscheinlichkeit eine etwas tiefere und plausiblere Begründung als in Lindenbergs Ansatz. Fasst man nämlich den Parameter $\beta$ selbst als Wahrscheinlichkeit auf, dann lässt sich $w_{j i}$ als eine Wahrscheinlichkeitsmischung des skalierten Erwartungsnutzens der Handlungsalternative $\left(v_{j i}\right)$ und des generellen Durchschnittsnutzens $(1 / n)$ interpretieren.

Betrachtet man schliesslich die Gleichung für die Wahlwahrscheinlichkeit unter inhaltlichen Gesichtspunkten, so ergibt sich bei geringem (hohem) Grad der situationalen Dominanz $\beta$ eine grosse (kleine) Bedeutung des Durchschnittsnutzens bei der Bestimmung der Auswahlwahrscheinlichkeit der $i$-ten Alternative. Eine stärkere Unsicherheit 
über das in der Entscheidungssituation letztlich dominierende Handlungsmotiv führt also tendenziell zu einer Betonung der durchschnittlichen Auszahlung bei der Handlungswahl. Die daher kleinere Gewichtung der Nutzenwerte der einzelnen Handlungsalternativen reflektiert die Ähnlichkeit der Präferenzen für verschiedene Handlungsorientierungen. Weil die Durchschnittsauszahlung für alle möglichen Handlungsmotive gleich ist, deutet dies gleichzeitig auf eine geringere Diskriminierungsleistung der aktuellen Handlungsorientierung hin.

\subsection{Wahl der Handlungsorientierung}

Zur Schliessung des Modells ist nun noch die Wahl der Handlungsorientierung zu formalisieren. Zur Modellierung des Lindenbergschen „frame switch" wird wiederum Colemans (1964) Hierarchieindex verwendet. Allerdings stehen nun die mit den verschiedenen Handlungsorientierungen einhergehenden Verteilungen der Wahlwahrscheinlichkeiten sowie deren Vergleich im Mittelpunkt. Genauer gesagt resultiert für jede Orientierung $j$ ein Grad der Differenzierung oder Diskriminierung in der jeweiligen Verteilung der (im obigen Sinne bestimmten) Wahlwahrscheinlichkeiten:

$\delta_{j}=H\left(w_{j}\right)=\frac{\ln n-F\left(w_{j}\right)}{\ln n}=1+\frac{\sum_{i} w_{j i} \ln w_{i j}}{\ln n}$ für alle $j$, so dass $0 \leq \delta_{j} \leq 1$, wenn die Konvention $w_{j i} \ln w_{j i}=$ 0 für $w_{j i}=0$ angewendet wird. Es gilt somit $\delta_{j}=0$, wenn $w_{j i}=(1 / n)$ für alle $i$ sowie $\delta_{j}=1$, falls $w_{j i}=1$ und $w_{j k}=0$ für alle $k \neq i$. Auf dieser Grundlage lässt sich Lindenbergs ,frame switching"-Hypothese präzisieren: Existieren Handlungsorientierungen mit verschiedenen Differenzierungsgraden $\delta_{1}, \delta_{2}, \ldots \delta_{m}$ der korrespondierenden Verteilungen der Wahlwahrscheinlichkeiten, so wird der Akteur jeweils diejenige Handlungsorientierung ersetzen, deren Differenzierungsgrad näher bei Null liegt. Anders ausgedrückt: Der Akteur wird diejenige Handlungsorientierung für seine Handlungsentscheidungen wählen, deren Diskriminationsgrad dem Wert Eins am nächsten kommt.

Dabei wird unterstellt, dass der Akteur die Diskriminierungsleistung einer gewählten Handlungsorientierung ständig überprüft und gegebenenfalls eine andere Orientierung wählt. Weil diese Annahme mit Lindenbergs Grundideen vereinbar scheint, aber dadurch eine enorme kognitive Leistungsfähigkeit des Akteurs vorausgesetzt wird, ist abschliessend eine kurze Diskussion der Deutung des DM als Modell für begrenzt rationales Verhalten erforderlich.

\section{Diskussion}

Die Formalisierung von Lindenbergs Grundideen zeigt, dass die kognitiven Anforderungen an einen Akteur, der im Sinne des DM entscheidet, über die Informationsverarbeitungskapazität hinausgehen, die für Entscheidungsträger im Erwartungsnutzenansatz unterstellt wird. Es ergeben sich daher Zweifel an einer Interpretation des DM als Modell für begrenzt rationale Verhaltensweisen, die kognitive Grenzen reflektieren: Zusätzlich zur Bestimmung der Erwartungsnutzen handelt ein typischer Akteur im Sinne des DM offenbar so, als ob er oder sie noch weitere „Berechnungen“ anstellt. Simon $(1990,1993)$ sieht in Wahrnehmungsmängeln und Informationsverarbeitungsproblemen die zentralen Hinderungsgründe für die Annahme rationalen Handelns im Sinne der Erwartungsnutzenmaximierung. Teilt man diese Einschätzung, so erscheint eine Interpretation des DM als Modell begrenzt rationalen Verhaltens nicht angemessen.

Dies bedeutet jedoch nicht, dass das DM überflüssig ist. Man kann das DM vielmehr als eine Erweiterung des traditionellen individualistischen Entscheidungskalküls betrachten. Rationales Verhalten im Sinne des Erwartungsnutzenmodells ergibt sich in der hier formalisierten Version von Lindenbergs Ansatz, wenn nur eine einzige Handlungsorientierung (z. B. zweckrationales Handeln) interessiert und daher vollständige Dominanz $(\beta=1)$ dieses Handlungsmotivs bei der Handlungsentscheidung besteht. Aus der Perspektive des reformulierten DM lässt sich der Grenzfall des Erwartungsnutzenansatzes damit als Modell eines „eindimensionalen" Akteurs im Sinne von Lüdemann/ Rothgang (1996) auffassen. Generell stellt das reformulierte DM aber eine Entscheidungstheorie dar, die in eher typischen Fällen $(\beta<1)$ nicht durch Lüdemann/Rothgangs Vorwurf des „eindimensionalen" Akteurs charakterisiert werden kann.

Ungeachtet der Interpretation des DM als mehr oder weniger allgemeine soziologische Verhaltenstheorie treten durch seine Reformulierung jedoch einige klärungsbedürftige Probleme in den Vordergrund. Zum einen ist fraglich, ob menschliche Akteure tatsächlich kognitive Fähigkeiten im Sinne des DM aufweisen. Dies gilt umso mehr, wenn man mit Simon bereits bezweifelt, dass die menschliche Informationsverarbeitungskapazität den geringeren kognitiven Anforderungen des Er- 
wartungsnutzenmodells gerecht wird. Kritisiert man also mit Simons Argumentation den Homo Oeconomicus der Wirtschaftstheorie, so scheinen erst recht Zweifel am Menschenbild des DM angebracht.

Unterstellt man dennoch Entscheidungsverhalten im Sinne des reformulierten DM, so trifft man damit die Annahme einer enormen Informationsverarbeitungskapazität. Dann aber stellt sich sofort die Frage, warum ein Akteur bei Handlungsentscheidungen nur eine einzige Handlungsorientierung verfolgt. Lindenberg begründet dieses Verhalten durch die Annahme kognitiver Beschränkungen. Im Zusammenhang mit dem ausgearbeiteten DM erscheint dieses Argument wenig zwingend und hilfreich, weil es mit den (für die Auswahl und Beibehaltung einer Handlungsorientierung) notwendigen Annahmen über die kognitiven Fähigkeiten kollidiert.

Wie kann man dennoch die Verfolgung eines einzigen Leitmotivs im reformulierten DM rechtfertigen? Ein mögliche Antwort liefert die Erkenntnis, dass verschiedene Handlungsorientierungen (z. B. Egoismus vs. Fairness) mit unterschiedlichen Wertvorstellungen und damit Nutzenfunktionen einhergehen. In einer Erweiterung des üblichen Kalküls wird die (für Handlungsentscheidungen relevante) Präferenzfunktion damit als Resultat einer vorherigen diskriminierenden Wahl zwischen verschiedenen Handlungsorientierungen (und damit unterschiedlichen Nutzenfunktionen) interpretiert. Die Festlegung auf eine einzige Handlungsorientierung oder Nutzenfunktion kann man dahingehend deuten, dass der Akteur eine eindeutige Bewertungsgrundlage benötigt, um seine oder ihre Entscheidungsfähigkeit zu gewährleisten. Eine einmal gewählte Nutzenfunktion wird unter sonst gleichen Bedingungen beibehalten, um überhaupt zu Verhaltensentscheidungen zu gelangen.

Aus dieser Perspektive bereichert das DM die traditionelle Entscheidungstheorie um die soziologisch relevante Dimension der Selektion des Handlungsleitmotivs. Allerdings stehen die dafür notwendigen Annahmen über die Aufnahme und Verarbeitung von Informationen dann im klaren Gegensatz zu seiner ursprünglichen Interpretation als Modell begrenzt rationalen Verhaltens.

\section{Literatur}

Coleman, J.S. 1964: Introduction to Mathematical Sociology. New York: Free Press.

Coleman J.S. 1990: Foundations of Social Theory. Cambridge: The Belknap Press of Harvard University Press.

Esser, H. 1993: The Rationality of Everyday Behavior: A Rational Choice Reconstruction of the Theory of Action by Alfred Schutz. Rationality and Society 5: 7-31.

Etzioni, A. 1994: Jenseits des Egoismus-Prinzips. Stuttgart: Schäffer-Poeschel.

Kreps, D.M. 1990: Game Theory and Economic Modelling. Oxford: Oxford University Press.

Lindenberg. S. 1993: Framing. Empirical Evidence, and Applications. S. 11-38 in Herder-Dornreich, P./Schenk, K.-E./Schmidtchen, D. (Hrsg.) Jahrbuch für Neue Politische Ökonomie Bd. 12, Neue Politische Ökonomie von Normen und Institutionen. Tubingen: Mohr.

Ludemann. C./Rothgang. H. 1996: Der "eindimensionale" Akteur: Eine Kritik der Framing-Modelle von Siegwart Lindenberg und Hartmut Esser. Zeitschrift fur Soziologie 25: 278-288.

Maasoumi, E. 1990: Information Theory. S. 101-117 in Eatwell, J./Milgate, M./Newman, P. (Hrsg.) The New Palgrave: Econometrics. New York: Norton.

Radner, R. 1980: Collusive Behavior in Noncooperative Epsilon-Equilibria of Oligopolies with Long but Finite Lives. Journal of Economic Theory 22: 136-154.

Selten, R. 1977: A Simple Game Model of Kidnapping. S. 139-155 in Henn, R./Moeschlin, O. (Hrsg.), Mathematical Economics and Game Theory. Essays in Honor of Oskar Morgenstern. Berlin: Springer.

Selten, R. 1990: Bounded Rationality. Journal of Institutional and Theoretical Economics 146: 649-658.

Simon, H.A. 1982: Models of Bounded Rationality. 2 vols. Cambridge: MIT Press.

Simon, H.A. 1990: Bounded Rationality. S. 15-18 in Eatwell, J./Milgate, M./Newman, P. (Hrsg.), The New Palgrave: Utility and Probability. New York: Norton.

Simon, H.A. 1993: Homo Rationalis: Die Vernunft im menschlichen Leben. Frankfurt a.M. und New York: Campus.

Theil, H. 1967: Economics and Information Theory. Amsterdam: North-Holland.

Tietz, R./Albers, W.Selten, R. (Hrsg.) 1988: Bounded Rational Behavior in Games and Markets. Berlin: Springer.

Weber, M. 1976 (1921): Wirtschaft und Gesellschaft. Grundriss der verstehenden Soziologie. Funfte Auflage. Tubingen: Mohr.

Williamson, O.E. 1985: The Economic Institutions of Capitalism. New York: Free Press. 